\section{Identification of the Human T Cell Lymphoma Virus in B Cell Lines Established from Patients with Adult T Cell Leukemia}

\author{
D. L. Mann, J. Clark, M. Clarke, M. Reitz, M. Popovic, \\ G. Franchini, C. D. Trainor, D. M. Strong, \\ W. A. Blattner, and R. C. Gallo \\ Laboratory of Human Carcinogenesis, Laboratory of Tumor Cell \\ Biology, and the Environmental Epidemiology Branch, National \\ Cancer-Institute, National Institutes of Health, and Department \\ of Surgery, Uniformed Services University for the Health \\ Sciences, Bethesda, Maryland 20205
}

A

bstract. Cell lines were established from the peripheral blood of two patients with adult $\mathrm{T}$ cell leukemia. In contrast to our previous experience, where all such lines expressed $\mathrm{T}$ cell markers, these two cell lines expressed B cell antigens and Ig light chains ( $\kappa$ on CF-2, $\lambda$ on HS). Human T cell lymphoma proviral (HTLV) sequences were demonstrated in both cell lines. Since only a portion of the cells in culture expressed Ig light chains, experiments were carried out to exclude the possibility that the cultures were not a mixture of $B$ and $T$ or non-B cells. Cells that expressed $\kappa$ - or $\lambda$-light chains were separated by cell sorting from $\kappa$ - or $\lambda$-negative cells and replaced in culture. Light chain negative cells reexpressed light chains after time in culture. After 5-azacytidine treatment of the cell lines, all cells expressed Ig light chains. These studies show that the human retrovirus HTLV, which has been demonstrated to be associated with certain $\mathrm{T}$ cell malignancies, can infect B cells or B cell precursors.

\section{Introduction}

The human $T$ cell lymphoma virus (HTLV) ${ }^{1}$ is a unique type $\mathrm{C}$ retrovirus that has been isolated from cells of patients with adult $T$ cell malignancies (1-5). After the initial isolation and identification of this virus by Gallo and colleagues $(1,2)$, Japanese investigators (6) identified a virus in Japanese patients with

Received for publication 6 December 1983 and in revised form 12 March 1984.

1. Abbreviations used in this paper: ATLA, acute T cell leukemia antigen; CBL, cord blood lymphocyte; EBNA, Epstein-Barr nuclear antigen; EBV, Epstein-Barr virus; HTLV, human T cell lymphoma virus; kb, kilobase; PBL, peripheral blood lymphocyte; TAC, anti-T cell growth factor receptor; TCGF, $\mathrm{T}$ cell growth factor.

The Journal of Clinical Investigation, Inc.

Volume 74, July 1984, 56-62 adult $\mathrm{T}$ cell leukemias which has proved thus far to be indistinguishable from HTLV (7). HTLV can be identified by the use of antibodies specific for the viral core (gag) proteins (8), by specific reverse transcriptase (9), and by nucleic acid hybridization (4).

Studies so far have indicated that this virus probably has trophism for $\mathrm{T}$ lymphocytes. This conclusion is based on cell surface markers on peripheral blood lymphocytes (PBLs) and tumor tissue from patients with HTLV infection (10-14). Cell lines established with lymphocytes from these patients demonstrated HTLV proviral replication and showed surface antigens characteristic of T cells (OKT3, OKT 11, and the sheep erythrocyte receptor, as well as TAC [anti-T cell growth factor receptor]) (12). Furthermore, cells cultured from these patients are representative of a subpopulation of lymphocytes: they are predominantly OKT4 positive and OKT8 negative. A minority of cells lines in tissue culture are of the OKT8 phenotype or of a double phenotype of OKT8 and OKT4 $(12,14,15)$. Cell lines are negative for surface Ig, Epstein-Barr virus (EBV), terminal deoxynucleotidyl transferase, and antigens characteristic of $B$ lymphocytes.

Further evidence that suggests that the virus infects primarily $T$ cells has been obtained in coculture experiments where umbilical cord blood lymphocytes (CBLs) have been infected with the HTLV. Cell lines established from the CBLs infected with HTLV express the OKT4 phenotype and other pan-T cell markers (15). Therefore, results so far indicate that the predominant infection of HTLV is in a T cell or, indeed, in a cell that can express $\mathrm{T}$ cell markers when placed in culture.

We now report the establishment of cell lines from two patients with classic adult $T$ cell leukemia and demonstrate that these cell lines have B cell characteristics and that the entire cell population is infected with the HTLV.

\section{Methods}

Cell culture. Cell lines under study were established from two patients with classic adult $T$ cell leukemia (16). 
Patient C.F. This patient, a 41-yr-old black male from Summerton, St. James, Jamaica, who moved to Miami, Florida, in 1972, was well until June 1981, when a diffuse erythematous scaly rash was proven by biopsy to be mycosis fungiodes. A hematologic workup at that time was reportedly normal, and he defaulted from methoxsalen, ultraviolet $\mathrm{A}$ photochemotherapy. 14 mo later, he presented with a 1-mo history of a 10-lb weight loss, anorexia, abdominal pain, and intermittent confusion. He was found to have diffuse adenopathy, bilateral pleural effusion, hepatosplenomegaly, a dry, scaly, nonpopular skin rash, and a markedly elevated calcium level. Skeleton $x$-rays were normal. The white blood count was 73,000 , with $70 \%$ pleiomorphic malignant lymphocytes with T cell markers (see below); lymph node biopsy, bone marrow, and lung biopsies, as well as pleural effusion cells, led to a diagnosis of large cell immunoblastic lymphoma with atypical features, as is characteristic of adult $T$ cell leukemia/lymphoma.

Patient H.S. This patient, a 20-yr-old black female from north central Florida, was well until January 1983, when she developed abdominal pain, nausea, vomiting, general malaise, an aching pain in her knees, and bilateral conjunctivitis. With the progression of these symptoms and the development of fever, diffuse adenopathy, and a persistent cough, she was admitted with a normal white count. $6 \mathrm{~d}$ later, her white count rose to 60,000 ; most of the cells were pleiomorphic and bore mature $T$ cell markers (see below). The patient had hypercalcemia and diffuse pulmonary infiltrates that consisted of nonspecific lymphocytic infiltration. Further staging documented diffuse mixed small and large cell lymphocytes in lymph nodes, skin, lung, bone marrow, and the central nervous system, as is consistent with adult $T$ cell leukemia/lymphoma.

PBLs from these patients were obtained and placed into culture by methods previously described (12). Cells were initially cultured in the presence of $20 \% \mathrm{~T}$ cell growth factor (TCGF). It was observed that the growth characteristics of these cells were different from those of the TCGF-dependent $T$ cell lines established from other patients. Furthermore, it was found that the cell lines grew in the absence of TCGF. Both cell lines were removed from TCGF and grown in 20\% fetal calf serum in modified Eagle's medium.

Cell surface antigen expression. To establish the fact that the cell lines were in fact from the representative patients, HLA typing was performed by the previously described methods $(17,18)$. Cell surface antigens were determined with the fluorescence-activated cell sorter. Antigen-saturating amounts of monoclonal antibodies (to be described) in $0.1 \mathrm{ml}$ were reacted with $10^{6}$ cells for $1 \mathrm{~h}$ at room temperature. The cells were washed and a fluorescein-labeled goat $\mathrm{F}\left(\mathrm{ab}^{\prime}\right)_{2}$ anti-mouse Ig antibody was added. After $1 \mathrm{~h}$ at $4^{\circ} \mathrm{C}$ the cells were washed and analyzed on the fluorescence-activated cell sorter (FACS-II; Becton-Dickinson FACS, Becton-Dickinson and Co., Sunnyvale, CA). The control myeloma protein (P3) was used in all experiments. All cells that demonstrated a forward light scatter pattern were analyzed, and the percentage of cells that showed fluorescence greater than that observed with the control myeloma protein were determined.

The following monoclonal antibodies were used in these experiments. Monoclonal antibodies to detect IgG, IgA, IgM, and the $\kappa$ - and $\lambda$-light chains were obtained from a commercial source. The monoclonal antibody 3 F10 detects a monomorphic determinant on HLA-A, B, and $C$ antigens. 3.1 detects a monomorphic determinant on HLA-DR antigens. The monoclonal antibody 4D12 detects a polymorphic determinant on some HLA-A and B allotypic antigens. This monoclonal antibody has been shown to react with a cell surface epitope that appears on HTLV-infected cells. The TAC antibody reacts with the receptor for TCGF. B1 detects an antigen on human B cells. OKT3 is a monoclonal antibody that detects normal peripheral blood $\mathrm{T}$ cells. The OKT4 and OKT8 antibodies, respectively, detect the helper/inducer phenotype and the cytotoxic/suppressor phenotype in normal PBLs.

EBV infection in these cell lines was tested for by assessing the presence of the Epstein-Barr nuclear antigen (EBNA) as described by Reedman and Klein (19).

Southern blot analysis. High molecular weight DNA was prepared from both the CF-2 and the HS cell lines. The DNA from the CF-2 cell lines was digested with the restriction endonucleases EcoRI or HindIII (Bethesda Research Laboratory, Gaithersburg, MD) in the buffer recommended by the supplier. The DNA from the HS cell line was digested with EcoRI or PstI. The digested DNA from both of these preparations was electrophoresed on $0.8 \%$ agarose and transferred to nitrocellulose filters. The filter with the CF-2 DNA digest was hybridized to a ${ }^{32} \mathrm{P}$ nick-translated HTLV probe, RU5, which contained the R and U5 sequences as previously described (20). The filters with the digested DNA from H.S. were hybridized to a nick-translated 23-3 probe, which is representative of the HTLV-1 genome (21).

\section{Results}

HLA typing of the cultured cell lines and the B cells isolated from PBLs were identical, demonstrating that the cultured cell lines were derived from the patients' PBLs. Both cell lines were $>90 \%$ positive for EBNA.

Comparison of the cell surface antigens on the PBLs and the cultured cell lines, as determined by monoclonal antibodies that detect $T$ cell-specific antigens, HLA, and Ig (isotypes, heavy and light chains), is shown in Table I. The PBLs from patient C.F. were $93 \%$ positive for the OKT3 antigen and demonstrated

Table I. Comparison of Cell Surface Antigens on PBLs and Cultured Cell Lines (CCL)

\begin{tabular}{|c|c|c|c|c|c|c|c|c|c|c|c|}
\hline \multirow[b]{2}{*}{ Cell source } & \multicolumn{11}{|c|}{$\%$ of cells reacting with antibody } \\
\hline & OKT3 & OKT4 & OKT8 & TAC & $3 \mathrm{~F} 10$ & 4D12 & B-1 & IgM & IgG & $\kappa$ & $\lambda$ \\
\hline PBL & 93 & 5 & 3 & 16 & 96 & 4 & 8 & 3 & 3 & 3 & 2 \\
\hline CCL & 0 & 0 & 0 & 31 & 92 & 44 & 60 & 3 & 0 & 50 & 0 \\
\hline \multicolumn{12}{|l|}{ H.S. } \\
\hline PBL & 55 & 39 & 1 & 36 & 93 & 28 & 23 & 2 & 2 & 33 & 3 \\
\hline CCL & 0 & 0 & 0 & 18 & 88 & 31 & 43 & 3 & 0 & 0 & 50 \\
\hline
\end{tabular}


low levels of reactivity with the OKT4 and OKT8 reagents. TAC antigen was found on $16 \%$ of the cells, which is the upper limit of levels of this antigen found on normal PBLs. The epitope detected by the 4D12 monoclonal antibody was low (4\%) on these PBLs. The total population of PBLs contained $\sim 8 \%$ B cells, as determined by the $B 1$ monoclonal antibody. Low levels of the Ig isotypes IgM and IgG were found on the cells, as were the light chains $\alpha$ and $\lambda$. In contrast, the cultured cell line from this patient had no $\mathrm{T}$ cell markers. $31 \%$ of these cells expressed the putative TCGF, as demonstrated by their reactivity to TAC. The cells maintained their expression of HLA-A, B, and C antigens, as demonstrated by the reactivity to the $3 \mathrm{~F} 10$ monoclonal antibody. $4 \mathrm{D} 12$ reacted with $44 \%$ of the cells. $60 \%$ of the cells were B1 positive. IgM was found on $3 \%$ of the cells, whereas $\kappa$ was represented on $50 \%$ of this particular cell line.

The cell surface antigen profile of the PBLs from H.S. was somewhat different from that of the cells obtained from C.F. OKT3 was represented on $55 \%$ of the cells, which is the lower limit of normal of the representation of this antigen on normal PBLs. OKT4 was found on $39 \%$ of the cells, whereas OKT8 was found on only $1 \%$. The determinant detected by the TAC antibody was found on $36 \%$ of the cells. HLA representation was the same as that found on normal PBLs. The epitope detected by the 4D12 monoclonal antibody was found on $28 \%$ of the cells. $23 \%$ of the cells appeared to be B cells, as determined by the monoclonal antibody B1. Low levels of IgM and IgG were found on the PBLs; however, the $k$-light chain was found on $33 \%$ of the PBLs. The $\lambda$-light chain was found on only $3 \%$ of these PBLs. As described above for C.F., the cultured cell line from this patient had no T cell markers. $18 \%$ of the cells expressed the antigen TAC. 31 and $43 \%$ of the cells, respectively, bore the 4D12 antigen and marker for B cells. IgM was found on $3 \%$ of the cells. $50 \%$ of the cells expressed $\lambda$-light chain at a level of $50 \%$. It is of interest that whereas the PBLs had determinants for the $\kappa$-light chain on the cell surface, this light chain was not detected in the cultured cell line.

The results suggested that a B cell line had been established from the PBLs of the above two patients. This assumption was based on the presence of a specific B cell antigen, B1, as well as on the expression of either the $\kappa$ - or $\lambda$-light chain. Since only $50 \%$ of the cells expressed the $k$-light chain and/or the $\lambda$-light chain in the respective cell cultures, it was possible that these cells were a mixed population, that is, $T$ cells (or null cells) and

Table II. Analysis of Surface Antigens on CF-2 Cells Sorted for $\mathrm{k}$-Light Chain

\begin{tabular}{llllllllll}
\hline \multirow{2}{*}{$\begin{array}{l}\text { Chain } \\
\text { sorted } \\
\text { for }\end{array}$} & $\begin{array}{l}\text { Time } \\
\text { post-sort }\end{array}$ & OKT3 & 3 F10 & 4D12 & 3.1 & TAC & IgM & $\kappa$ \\
\cline { 3 - 9 }$\kappa-$ & $24 \mathrm{~h}$ & 0 & 89 & 4 & 15 & 6 & 0 & 4 \\
$\kappa+$ & $24 \mathrm{~h}$ & 0 & 92 & 86 & 78 & 72 & 10 & 89 \\
$\kappa-$ & $5 \mathrm{~d}$ & 0 & 96 & 31 & 39 & 30 & 0 & 36 \\
$\kappa-$ & $2 \mathrm{wk}$ & 0 & 94 & 36 & 29 & 35 & 0 & 40 \\
\hline
\end{tabular}

Table III. Analysis of Cell Surface Antigens on HS Cells Sorted for $\lambda$-Light Chain

\begin{tabular}{llllllllll}
\hline \multirow{2}{*}{$\begin{array}{l}\text { Chain } \\
\text { sorted } \\
\text { for }\end{array}$} & $\begin{array}{l}\text { Time } \\
\text { post-sort }\end{array}$ & OKT3 & 3 F10 & 4D12 & 3.1 & TAC & $\lambda$ & IgM \\
\cline { 3 - 9 } & Of cells reacting with antibody \\
$\lambda-$ & $24 \mathrm{~h}$ & 0 & 92 & 2 & 21 & 5 & 2 & 0 \\
$\lambda+$ & $24 \mathrm{~h}$ & 0 & 94 & 84 & 67 & 87 & 89 & 0 \\
$\lambda-$ & $5 \mathrm{~d}$ & 0 & 96 & 26 & 34 & 24 & 26 & 0 \\
$\lambda-$ & 2 wk & 0 & 97 & 22 & 29 & 26 & 22 & 0 \\
\hline
\end{tabular}

B cells. To determine if two populations of cells existed in the cell culture, fluorescence cell sorting was performed. Sorting was done on both of the cell lines; labeling was done with the reagent to detect the $\kappa$-light chain on the CF-2 cells and the $\lambda$-light chain on the HS cells. $10 \%$ of the cells that demonstrated the greatest degree of positivity to either of these light chains were recovered with the sort, as were all of those cells that showed no fluorescence with the reagent to detect these two determinants. The positive and negative cells were recovered and placed back into culture. These cells were then analyzed for cell surface determinants $24 \mathrm{~h}$ post-sort, $5 \mathrm{~d}$ post-sort, and 2 wk post-sort. The results of the cell surface analysis of the cells obtained from the sort are shown in Tables II and III. 24 $h$ post-sort, the representation of HLA-A, B, and C as detected by the 3 F 10 monoclonal antibody was nearly identical in both the $\kappa$-positive and $\lambda$-negative sorted cells. $4 \%$ of the $\kappa$-negative cells expressed the $\kappa$-light chain and no IgM was detected. The TAC antigen was detected on $6 \%$ of the cells, the epitope was detected by the 4D12 monoclonal antibody on 4\%, and HLADR was found on $15 \%$. Those cells that were sorted for $\kappa$-positivity showed $89 \% \kappa$-light chain, $10 \%$ IgM, 72\% TAC, $86 \%$ 4D12 positivity, and $78 \%$ reaction with the 3.1 monoclonal antibody to detect HLA-DR. At $5 \mathrm{~d}$ and 2 wk post-sort, the $\kappa$-negative cell in culture showed a distribution of the cell surface determinants not unlike that seen in the original culture. An almost identical result was seen with the HS cell line sorted for the $\lambda$-light chain (Table III). Again, the cells that were $\lambda$-negative at $24 \mathrm{~h}$ post-sort showed low levels of $\lambda$-light chain, TAC, and 4D12. HLA-DR was expressed on $21 \%$ of the cells. The majority of the cells that were $\lambda$-positive expressed the 4D12 antigen, HLA-DR, TAC, and the $\lambda$-light chain. The cultured $\lambda$-negative cells were analyzed $5 \mathrm{~d}$ and $2 \mathrm{wk}$ post-sort. The antigenic profile at these times in culture was essentially the same as that seen in the original cultured cell line, with representation of the $\lambda$ light chain on 26 and $22 \%$ of the cells, respectively; TAC, 24 and 26\%; HLA-DR, 34 and 29\%; and 4D12, 26 and 22\%. Both the sorted $\kappa$-positive cells from C.F. and the $\lambda$-positive cells from H.S. were viable $24 \mathrm{~h}$ post-sort; however, these cells were dead (as determined by trypan blue dye exclusion) after $5 \mathrm{~d}$ in culture. Three separate attempts were made to grow the $\kappa$-positive cells post-sort. These attempts failed. However, when supernatants 
from the light chain negative cultures were added to the $\kappa$ - or $\lambda$-positive cells, viability and growth were observed.

Since both the $\kappa$-negative cells and the $\lambda$-negative cells expressed (after $2 \mathrm{wk}$ in culture) similar antigenic profiles to the original cell line, we repeated the cell-sorting experiment on both of these cell lines. The CF-2 cells were sorted for $k$-light chain on three successive occasions after the $\kappa$-negative cells were in culture for $2 \mathrm{wk}$. The experiments recapitulated the results that are shown in Tables II and III, where the $k$-negative cells again became positive for $\alpha$-light chain within 2 wk of separating the light chain-positive from the light chain-negative cells. One interpretation of these results is that the expression of both the $\kappa$ - and $\lambda$-light chains, as well as of the other cell surface determinants-TAC, HLA-DR, and the 4D12 epitopeis a manifestation of cell maturation. Furthermore, the results strongly suggest that this is a uniform cell population that shows antigenic determinants based on the stages of maturation of the cell line, the most mature cell being that which expresses the light chain and the other antigenic determinants.

To examine further the question of whether all cells could express the antigens, cells were examined for cell surface antigen expression after treatment with 5-azacytidine. Figs. 1 and 2 show the results of analysis of the $\kappa$ - and $\lambda$-light chain expression in the respective cell lines. As can be seen from these figures, most, if not all, of the cells expressed $\alpha$ - or $\lambda$-light chain after treatment with 5 -azacytidine. The cells were analyzed for other cell surface markers. TAC, 4D12, and HLA-DR antigens increased in expression to the same extent as did the light chain determinant (data not shown).

Figs. 3 and 4 show the results of Southern blot analyses of restriction endonuclease digests of DNA from both the CF-2

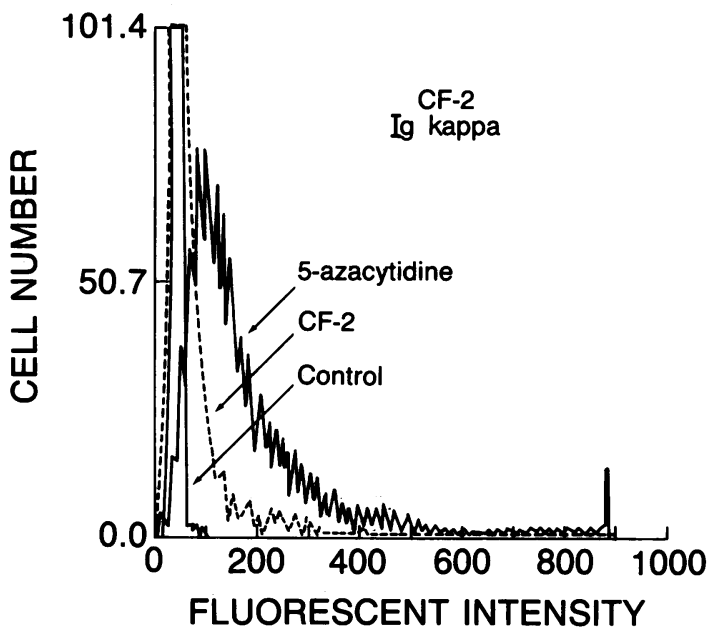

Figure 1. Analysis of cell surface Ig $\kappa$-light chains on cell line CF-2. Control fluorescence developed with P-3 mylotonin protein and fluorescein-labeled goat anti-mouse Ig. This figure shows the increase in expression of $\alpha$-light chains after the cells were treated with 5-azacytidine.

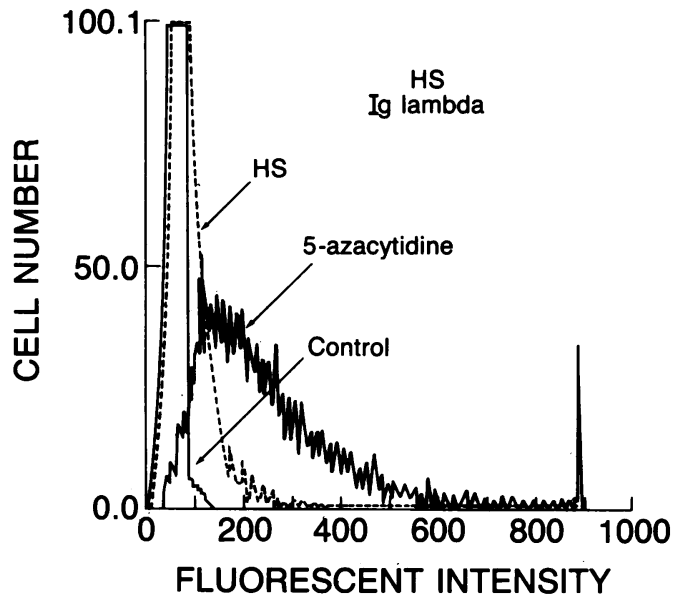

Figure 2. Analysis of cell surface Ig $\lambda$-light chains on cell line HS. Control fluorescence developed with P-3 myeloma protein and fluorescein-labeled anti-mouse Ig. This figure shows the increase in $\lambda$-light chain expression after the cells were treated with 5 -azacytidine.

and HS cell lines. Fig. 3 shows the results from the CF-2 cell line. Lane $a$ shows the blot obtained when the CF-2 DNA was digested with the EcoRI endonuclease and reveals a single band at 22 kilobases $(\mathrm{kb})$. Lane $b$ shows the results of the digest of the CF-2 DNA with HindIII endonuclease. Three bands can be readily identified at $5.7,4.3$, and $2.8 \mathrm{~kb}$. The results indicate that there is a single copy of the integrated proviral DNA in this cell line and that the provirus is clonal with respect to the integration site. The results of Southern blot analysis of the DNA obtained from H.S. are shown in Fig. 4. The EcoRI digest did not show a discrete viral band, whereas the PstI digest showed

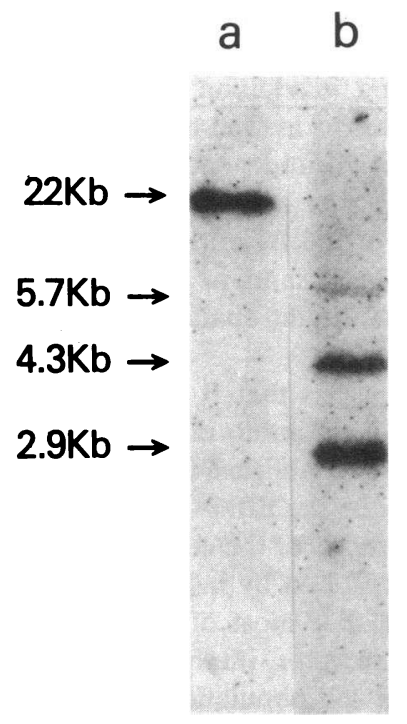

Figure 3. Southern blots of high molecular weight DNA from cell line CF-2. DNA was digested with restriction endonucleases EcoRI (lane $a$ ) and HindIII (lane $b$ ) and hybridized to the ${ }^{32} \mathrm{P}$ nick-translated RU5 probe. The arrows indicate the size of the digested fragments in kilobases calculated from a standard curve using HindIII digested phage DNA. 


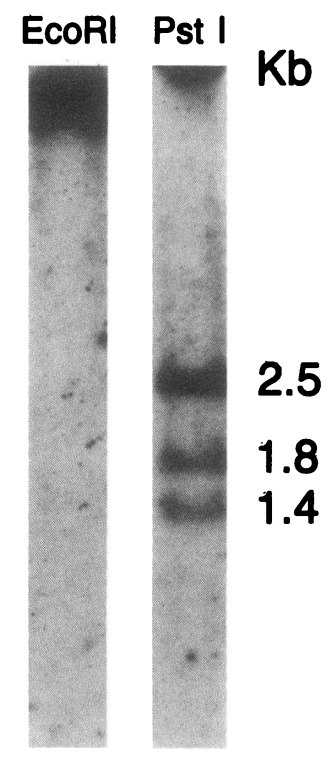

Figure 4. Southern blots of high molecular weight DNA from cell line HS. DNA wàs digested with restriction endonuclease EcoRI and PstI and hybridized to a ${ }^{32} \mathrm{P}$ nick-translated HTLV probe, $\lambda 23-3$.

bands at $2.5,1.8$, and $1.4 \mathrm{~kb}$. These bands represent the internal restriction fragments of the HTLV genome as determined by blotting with the $\lambda 23-3$ probe. The results demonstrate that most of the cells in both of these cell cultures were infected with HTLV and that the CF-2 cell line represents the expansion of a single cell infected by HTLV, whereas HS cells are the expansion of several cells that have HTLV integrated in different regions of the DNA.

\section{Discussion}

Cell lines that were growing independently of TCGF were established from two patients with clinically defined adult $\mathrm{T}$ cell leukemia. Neither cell line demonstrated the markers usually associated with normal $\mathrm{T}$ cells or those markers found on cells cultured from other patients with adult $\mathrm{T}$ cell leukemia. Such cultures are predominantly $T$ cells and, in addition, demonstrate the expression of the HLA-DR antigens as well as high levels of the TCGF receptor. Both of the TCGF-independent cell lines established from the two patients under study had relatively high levels of the TCGF receptor, of the B1 antigen which is found on normal peripheral blood B cells, and, in one instance, of the $k$-light chain (CF-2) and the $\lambda$-light chain on the cell line HS.

There is a single report that suggests that B lymphocytes may be infected with HTLV (21). Yamamoto et al. reported the results of studies on seven cell lines established from patients with adult $T$ cell leukemia that express the EBNA. In addition to the expression of the EBNA, up to 7\% of the cells expressed the acute $\mathrm{T}$ cell leukemia antigen (ATLA) by indirect immunofluorescence. They also reported that most of the cells in culture were negative for E-rosette receptor, implying that the cell cultures were a mixed $\mathrm{T}$ and $\mathrm{B}$ cell population. To dem- onstrate that the B cells were in fact infected with the HTLV, two of the cell lines were cloned. Cloning efficiencies range from 0.1 to $3.1 \%$ in the two cell lines. Clones established from these cell lines showed variable expression of ATLA, and again most of the cells expressed EBNA. Because the cloning efficiency was extremely low and not the entire cell population expressed the ATLA, the authors did not demonstrate decisively that the cells in the culture that were infected were in fact B cells and that the cell populations tested were not a mixture of $\mathrm{T}$ and $\mathrm{B}$ cells.

We carried out studies designed to demonstrate that cells in the culture that expressed light chains accounted for the HTLV infection that was detected by Southern blot analysis. Analysis of the cell surface markers on cells sorted for their respective light chains, subsequent culture with demonstration of reexpression of light chains on the negative cells, as well as the studies with 5-azacytidine-treated cells clearly indicate that a single population of cells was established in culture and that these cells were of a B cell lineage. Another piece of evidence that supports the contention that the cells are in fact B cells, or at least of early B cell lineage, is the observation that both cell lines were positive for the EBNA.

We conclude from these results that the cell lines established from the patients with classic $T$ cell leukemia were $B$ cells or $B$ cell precursors that matured in culture. The question remains as to whether or not the $T$ cells from these patients are also infected. Some preliminary results suggest that this is so. In Southern blot analysis of the DNA prepared from PBLs from the patient C.F. (93\% OKT3 positive, Table I), three different clonally integrated proviruses were seen. This indicates that at least three independent infections occurred in this population of peripheral blood $\mathrm{T}$ cells. Thus, the possibility exists that the malignant cell is in fact the $T$ cell and that the infected $B$ cell is the result of an incidental infection that did not result in malignant transformation.

A possible explanation for the in vitro growth of B cells rather than $T$ cells from these patients may be that infection of the same cell with EBV and HTLV confers a growth advantage. Markham et al. (22) reported that they were able to increase the percentage of human EBV-positive B cell lines established in culture by infecting with primate retroviruses, Gibbon ape leukemia virus, and the simian sarcoma virus. Further studies of these cell lines demonstrated a restricted expression of the provirus and of their proteins in these B cell lines (23). This is not unlike the observations made with the HTLV cell lines. Neither of the cell lines produced detectable gag proteins. The CF-2 cell line did show faint bands with Northern blotting, indicating low levels of production of proviral RNA as well as low levels of positivity in in vitro hybridization studies. These results contrast with those from the cell lines that can be routinely established from patients with HTLV-associated neoplasia. These cell lines have $\mathrm{T}$ cell markers, and products of the provirus can readily be detected. In addition, virus production by the $T$ cell lines has been well documented, whereas virus production thus far has not been detected in the B cell lines. These different 
properties of the $T$ cells and $B$ cells provide materials to study the control of HTLV production at the molecular level.

An unusual feature of these B cell lines is the expression of the cell surface marker for the TCGF receptor, TAC. The role of this receptor and the relationship to HTLV infection are not well understood. All T cell lines established from patients with HTLV-associated neoplasia express this determinant at high levels. It was originally thought that this indicated a cell growth dependence on exogenous or endogenous production of TCGF. However, more recent studies demonstrated a lack of dependence of some cell lines on exogenous TCGF; this suggests that the determinant detected by the TAC antibody may be a product of HTLV infection and replication. These relationships are now under study. The B cell lines established from these patients underscore the independence of TAC expression and TCGF dependence. The addition of TCGF to the cultures did not change the growth characteristics or the expression of cell surface markers in these cells (data not shown).

There is a suggestion, however, that a growth factor is involved in the maturation of the cells. Both cell lines, when sorted for $\kappa$ - or $\lambda$-light chains and placed in culture, did not grow, and within a very short time no viable cells could be seen in these cultures. The reversal of this process with supernatants from the growing culture, while not directly demonstrated, indicates that a growth factor may be involved in cell replication. The possibility that a factor produced by the cell itself is involved in the maturation of the cell and affects the continuation of growth of this cell line is under investigation.

The results of these studies demonstrated conclusively that the human $T$ cell lymphoma virus can in fact be found in B cells of patients with a $T$ cell malignancy. Since it is obvious that these patients had a $\mathrm{T}$ cell disease, the question arises as to why only $\mathrm{T}$ cells undergo malignant transformation with infection with this virus. One plausible explanation is that the virus infects an undifferentiated stem cell. Under appropriate stimulation (such as growth factors), malignant transformation occurs. It is of interest in this regard that newborn CBLs are relatively easy to infect whereas adult PBLs are not. The cells that grow and manifest infection in the CBLs have the OKT4 phenotype, which recapitulates the phenotype of infected leukemic cells. Adult PBLs have ample cells with the OKT4 phenotype that could potentially be infected. Thus, one can conclude that the virus does not necessarily infect cells with this phenotype. Rather, CBLs contain stem cells not present in adult PBLs that are infected and that express the OKT4 antigen as a result of the expansion of this cell population under the influence of TCGF, a factor necessary to establish infected cell lines from CBLs. One might predict that infection might occur in a cell that shows a B cell phenotype if these experiments were conducted with B cell growth factor rather than TCGF. This question is under investigation.

Using PBLs ( $T$ cells) from these individuals as well as from the $B$ cell line may give clues as to the transforming events that occur in a cell that is infected with this retrovirus, as well as allow the exploration of growth factors and their importance in this malignancy.

\section{References}

1. Poiesz, B. J., F. W. Ruscetti, A. F. Gazdar, P. A. Bunn, J. D. Minna, and R. C. Gallo. 1980. Isolation of type-C retrovirus particles from cultured and fresh lymphocytes of a patient with cutaneous T-cell lymphoma. Proc. Natl. Acad. Sci. USA. 77:7415-7519.

2. Poiesz, B. J., F. W. Ruscetti, M. S. Reitz, V. S. Kalyanaraman, and R. C. Gallo. 1981. Isolation of a new type C retrovirus (HTLV) in primary uncultured cells of a patient with Sezary T-cell leukaemia. Nature (Lond.). 294:268-271.

3. Reitz, M. S., Jr., V. S. Kalyanaraman, M. Robert-Guroff, M. Popovic, M. G. Sarnagadharan, P. S. Sarin, and R. C. Gallo. 1983. Human T-cell leukemia/lymphoma virus: the retrovirus of adult T-cell leukemia/lymphoma. J. Infect. Dis. 147:399-405.

4. Reitz, M. S., B. J. Poiesz, F. W. Ruscetti, and R. C. Gallo. 1981. Characterization and distribution of nucleic acid sequences of a novel type $\mathrm{C}$ retrovirus isolated from neoplastic human T lymphocytes. Proc. Natl. Acad. Sci. USA. 78:1887-1891.

5. Reitz, M. S., Jr., M. Popovic, B. F. Haynes, S. C. Clark, and R. C. Gallo. 1983. Relatedness by nucleic acid hybridization of new isolates of human T-cell leukemia-lymphoma virus (HTLV) and demonstration of provirus in uncultured leukemic blood cells. Virology. 126:688-692.

6. Yoshida, M., I. Miyoshi, and Y. Hinuma. 1979. Isolation and characterization of retrovirus from cell lines of human adult T-cell leukemia and its implication in the disease. Proc. Natl. Acad. Sci. USA. 79:2031-2035.

7. Popovic, M., M. S. Reitz, Jr., M. G. Sarngadharan, M. RobertGuroff, V. S. Kalyanaraman, Y. Nakao, I. Miyoshi, J. Minowada, M. Yoshida, Y. Ito, and R. C. Gallo. 1982. The virus of Japanese adult T-cell leukaemia is a member of the human T-cell leukaemia virus group. Nature (Lond.). 300:63-66.

8. Kalyanaraman, V. S., M. G. Sarngadharan, B. J. Poiesz, F. W. Ruscetti, and R. C. Gallo. 1981. Immunological properties of a type C retrovirus isolated from cultured human T-lymphoma cells and comparison to other mammalian retroviruses. J. Virol. 38:906-913.

9. Rho, J. M., B. J. Poiesz, F. W. Ruscetti, and R. C. Gallo. 1981. Characterization of the reverse transcriptase from a new retrovirus (HTLV) produced by a human cutaneous T-cell lymphoma cell line. Virology. 112:355-358.

10. Gallo, R. C., V. S. Kalyanaraman, M. G. Sarngadharan, A. Sliski, E. C. Vonderheid, M. Maeda, Y. Nakao, K. Yamada, Y. Ito, N. Gutensohn, S. Murphy, P. A. Bunn, Jr., D. Catovsky, M. F. Greaves, D. W. Blayney, W. Blattner, W. F. H. Jarrett, H. zur Hausen, M. Seligmann, J. C. Brouet, B. F. Haynes, B. V. Jegasothy, E. Jaffe, J. Cossman, S. Broder, R. I. Fisher, D. W. Golde, and M. Robert-Guroff. 1983. The human type-C retrovirus: association with a subset of adult T-cell malignancies. Cancer Res. 43:3829-3845.

11. Gallo, R. C., D. Mann, S. Broder, F. W. Ruscetti, M. Maeda, V. S. Kalyanaraman, M. Robert-Guroff, and M. S. Reitz. 1982. Human T-cell leukemia-lymphoma virus (HTLV) is in T- but not B-lymphocytes from a patient with cutaneous T-cell lymphoma. Proc. Natl. Acad. Sci. USA. 79:4680-4683.

12. Popovic, M., P. Sarin, M. Robert-Guroff, V. S. Kalyanaraman, D. Mann, J. Minowada, and R. C. Gallo. 1983. Isolation and transmission 
of human retrovirus (human T-cell leukemia virus). Science (Wash. DC). 219:856-859.

13. Huttari, T., T. Uchiyama, T. Toibana, K. Takasuki, and $H$ Uchino. 1981. Surface phenotype of Japanese adult T-cell leukemia cells characterized by monoclonal antibodies. Blood. 58:645-647.

14. Popovic, M., G. Lange-Wentzin, P. S. Sarin, D. Mann, and R. C. Gallo. 1983. Transformation of human umbilical cord blood Tcell leukemia/lymphoma virus (HTLV). Proc. Natl. Acad. Sci. USA. 80:5402-5406.

15. Mann, D. L., M. Popovic, C. Murray, C. Neuland, D. M. Strong, P. S. Sarin, R. C. Gallo, and W. A. Blattner. 1983. Cell surface antigen expression in newborn cord blood lymphocytes infected with HTLV. J. Immunol. 131:2021-2026.

16. Blayney, D. W., E. S. Jaffe, R. I. Fisher, G. P. Schechter, J. Cossman, M. Robert-Guroff, V. S. Kalyanaraman, W. A. Blattner, and R. C. Gallo. 1983. The human T-cell leukemia/lymphoma virus lymphoma lytic bone lesions and hypercalcemia. Ann. Intern. Med. 93:144151.

17. Amos, D. B., P. Pool, and J. Frier. 1980. HLA-A, HLA-B, HLA-C, and HLA-DR. In Manual of Clinical Immunology (second edition). N. R. Rose and H. Friedman, editors. American Society of Microbiology, Washington, DC. 978-986.

18. Mann, D. L., L. Abelson, P. Henkart, S. Harris, and D. B. Amos. 1975. Serologic detection of B lymphocyte antigens. In Histocompatibility
Testing. F. Kissmeyer-Neilson, editor. Munksgaard, Copenhagen. 705707.

19. Reedman, B., and G. Klein. 1973. Cellular localization of an Epstein-Barr virus-associated couplement fixing antigen in producer and non-producer lymphoblastoid cell lines. Int. J. Cancer. 11:499-520.

20. Manzari, V., F. Wong-Staal, G. Franchini, S. Colombini, E. P. Gelmann, S. Oroszlan, S. P. Staal, and R. C. Gallo. 1983. Human T-cell leukemia-lymphoma virus, HTLV: molecular cloning of an integrated defective provirus and flanking cellular sequences. Proc. Natl. Acad. Sci. USA. 80:1574-1578.

21. Yamamato, N., T. Matsumoto, Y. Kayanagi, Y. Tanaka, and Y. Hinuma. 1982. Unique cell lines harbouring both Epstein-Barr virus and adult T-cell leukemia virus, established from leukaemia patients. Nature (Lond.). 299:367-369.

22. Markham, P. D., F. Ruscetti, S. Z. Salahuddin, R. E. Gallagher, and R. C. Gallo. 1979. Enhanced induction of growth of B lymphoblasts from fresh human blood by primate type-C retroviruses, Gibbon ape leukemia virus and simian sarcoma virus. Int. J. Cancer. 23:148-156.

23. Markham, P. D., F. W. Ruscetti, V. S. Kalyanaraman, L. Ceccherini-Nelli, N. R. Miller, M. S. Reitz, S. Z. Salahuddin, and R. C. Gallo. 1981. Restricted expression of retrovirus nucleic acids and proteins in primate type $C$ virus (Gibbon ape leukemia-simian sarcoma virus)initiated human B-lymphoblastoid culture. Cancer Res. 41:2738-2744. 